\title{
EXPOSICIÓN A CAMPOS ELECTROMAGNÉTICOS EN UN SERVICIO DE REHABILITACIÓN
}

\author{
ACCÉSIT POSTERS. IV JORNADAS NACIONALES DE LOS SERVICIOS DE PREVENCIÓN \\ DE RIESGOS LABORALES EN EL ÁMBITO SANITARIO, 16-17 DE NOVIEMBRE DE 2006. \\ HOSPITAL 12 DE OCTUBRE. MADRID.
}

\author{
CONSUELO RODRIGO GARCÍA-PANDO, JUAN JOSÉ GRANADOS ARROYO, \\ PURIFICACIÓN MOZO BLANCO, RAFAEL FURIOS RESA, Y CRISTINA GOENAGA OLAIZOLA.
}

Servicio de Prevención de Riesgos Laborales. Área 9 (SERMAS). Leganés. Madrid.

\section{RESUMEN}

Los Campos Electromagnéticos están presentes en diferentes puestos de trabajo dentro del ámbito sanitario. Se han realizado miles de estudios en las últimas décadas tratando de conocer los efectos perjudiciales que la exposición a ellos pueda suponer para la salud de los trabajadores. La publicación de la Directiva 2004/40/CE de 29 de abril de 2004, del Parlamento Europeo y del Consejo, que establece las disposiciones mínimas de seguridad y de salud relativas a la exposición de los trabajadores a los riesgos derivados de los agentes físicos y una actualización bibliográfica de los posibles efectos adversos de los campos electromagnéticos, nos sirve como marco de referencia para conocer la situación que nos encontramos en un servicio de Rehabilitación.

Con los niveles de exposición medidos en este servicio y los conocimientos existentes en el momento actual sobre efectos biológicos de los CEM, no se detectan exposiciones que supongan riesgo para la salud de sus trabajadores.

PALABRAS CLAVE: Campos electromagnéticos (CEM). Efectos biológicos. Comisión Internacional sobre Radiaciones No Ionizantes (ICNIRP).

\section{SUMMARY}

Electromagnetic Fields (EMF) are present in different workplaces inside the sanitary area. Thousands of studies have been realized in the last decades trying to establish the harmful effects that exposure to them can suppose for the health of workers. The publication of the Directive 2004/40/CE of 29 April 2004, of the European Parliament and of the Council, which establishes the minimal health and safety requirements regarding the exposure of workers to the risks arising from physical agents and a bibliographical update of the possible adverse effects of electromagnetic fields, assists us as a frame of reference to know the situation that we are in as a service of Rehabilitation.

With the levels of exposure measured in this service and existing knowledge at the current time on the biological effects of the EMF, no exposures are detected that might suppose a risk for workers' health.

KEY WORDS: Electromagnetic fields (EMF). Biological effects. International Comission on Non Ionizing Radiation Protection (ICNIRP). 


\section{INTRODUCCIÓN}

La Directiva 2004/40/CE de 29 de abril de 2004, del Parlamento Europeo y del Consejo ${ }^{(1)}$ considera como Campos Electromagnéticos (CEM), a los campos magnéticos estáticos y los campos eléctricos, magnéticos y electromagnéticos variables en el tiempo, de frecuencias entre 0 y $300 \mathrm{GHz}$. Son radiaciones NO IONIZANTES

La exposición a CEM en el Servicio de Rehabilitación la podemos encontrar en:

- Electro estimulación neuromuscular transcutánea (TENS):

- High tens: $50-100 \mathrm{~Hz} / 0-100 \mathrm{~mA}$

- Low tens: 2-5 Hz/mayor de $100 \mathrm{~mA}$.

- Magnetoterapia utiliza CEM variables de forma pulsada (CEMP) con baja frecuencia $(1-100 \mathrm{~Hz})$ e intensidad ( 1 - 100 gauss).

- Ondacorta: utiliza CEM de 27,12MHz

- Microondas: utiliza CEM de $2450 \mathrm{MHz}$

Nuestro objetivo es una actualización sobre efectos en la salud para los trabajadores expuestos a campos electromagnéticos en un servicio de rehabilitación.

\section{MATERIAL Y MÉTODOS}

Se realizó una revisión de las publicaciones realizadas hasta la fecha referidas a campos electromagnéticos y efectos para la salud ${ }^{(2,3,4,5,6,7,8,9)}$. También se analizaron los posibles CEM que pueden encontrarse en un Servicio de Rehabilitación ${ }^{(10)}$ y se realizaron determinadas mediciones CEM en este Servicio.

\section{RESULTADOS}

La Comisión Internacional para la Protección de Radiaciones No Ionizantes estableció los siguientes mecanismos de interacción de los CEM con los sistemas biológicos.

Efectos biológicos de los CEM en función de su frecuencia (ICNIRP.2004):

- < $100 \mathrm{KHz}$ : inducción de corrientes débiles o estimulación de membranas.

- $100 \mathrm{KHz}-10 \mathrm{MHz}$ : transición de preponderancia desde corrientes inducidas a la producción de calor.

- $10 \mathrm{MHz}$ - $300 \mathrm{GHz}$ : producción de calor por inducción de corrientes intensas
Otros modelos de interacción como afectar al ritmo de reacciones químicas donde intervengan radicales libres o mecanismos de resonancia o amplificación de señales que hicieran a las células especialmente sensibles a los campos, son solo teorías en estudio pendientes de confirmación.

Se han descrito diferentes efectos adversos como los referidos a la corriente de contacto que, cuando es de gran intensidad, puede causar desde pequeños estímulos musculares o nerviosos a graves arritmias e incluso la muerte.

No se ha podido demostrar evidencia de que los CEM causen "Hipersensibilidad Electromagnética" (Workshop on Electrical Hypersensitivity, WHO 2005).

Aunque se han encontrado algunas alteraciones que deben ser estudiadas (Reflex Project, 2004)., no hay constancia de que la exposición a los campos electromagnéticos no ionizantes cause directamente daño en las moléculas de los seres vivos, y en particular en su ADN.

\section{EFECTOS ADVERSOS DE CEM DE BAJA FRECUENCIA $(<100$ KHZ)}

- Dos grandes "pooled análisis" Greenland et al, 2000 (12 estudios) y Ahlbom et al, 2000 (9 estudios) llegaron a conclusiones son similares: hay una evidencia débil - RR 2- de leucemia en niños para exposiciones superiores a $0,3 \mu \mathrm{T}$. Sin embargo, solo unos pocos niños tenían estos niveles, lo que resta fuerza a la asociación.

- En base a esto la IARC (Agencia Internacional de Investigación del Cáncer) en 2002 consideró a los campos magnéticos de baja frecuencia como posible carcinógeno humano (riesgo escasamente probado en las personas e insuficientemente probado en experimentos con animales).

- No se ha visto relación con cualquier otro tipo de cáncer.

- No existe evidencia de efectos consistentes en estudios con hormonas, sistema inmune, química sanguínea, estudios de laboratorio en células o estudios de laboratorio en animales

- Tampoco en defectos al nacimiento, alteración de la función del sistema inmune, reproducción, desarrollo o aprendizaje. 


\section{EFECTOS ADVERSOS DE CEM ENTRE 100 KHZ Y 10 GHZ (MICROONDAS Y ONDA CORTA):}

- Por el calor: inducción de cataratas en los ojos, varias respuestas fisiológicas y termo-reguladoras, teratogenicidad.

- Frecuencias entre aproximadamente $200 \mathrm{MHz}$ y $6.5 \mathrm{GHz}$, en personas que oyen normal, han percibido pulsaciones de campos.

- Ninguna acumulación de daño ocurre en tejidos que han sido expuestos repetidamente a bajos niveles de RF.

- Estudios (Larsen et al 1991, Oullet-Hellstrom and Stewart 1993, Taskinen et al 1990) en fisio- terapeutas expuestos por las terapias de diatermia onda corta $(27,12 \mathrm{MHz})$ o microondas han encontrado alteraciones en la fertilidad o aumento de abortos, pero factores de confusión y sesgos les restan fiabilidad. Revisados por Verschaeve and Maes 1998, Heynick and Merrit 2003 and Feychting, 2005, no han encontrado efectos adversos. Con los estudios disponibles en la actualidad los efectos reproductivos no entrañan riesgo para la salud a niveles por debajo de las recomendaciones en vigor.

En febrero de 2005 la empresa Helios Electromedicina S.L. realizó las mediciones de densidad de potencia que se exponen en la tabla I y que se encuentran en niveles inferiores a los valores que dan lugar a una acción establecidos por la ICNIRP y recogidos en la Directiva 2004/40/CE.

Tabla 1. Medidas de 9 equipos de microondas (2.450 MHz) con potencia de salida de $250 \mathrm{~W}$ y tomando siempre la medición más defavorable.

\begin{tabular}{|l|c|c|c|c|}
\hline Punto de medida & $\begin{array}{c}\text { Radiación } \\
\text { media } \\
\left(\mathrm{mW} / \mathrm{cm}^{2}\right)\end{array}$ & $\begin{array}{c}\text { Rango } \\
\left(\mathrm{mW} / \mathrm{cm}^{2}\right)\end{array}$ & $\begin{array}{c}\text { Radiación máxima } \\
\text { permitida } \\
\left.\mathrm{mW} / \mathrm{cm}^{2}\right) \\
\text { UNE 20-613-88 }\end{array}$ & $\begin{array}{c}\text { Valores que dan } \\
\text { lugar a una acción } \\
\left(\mathrm{W} / \mathrm{m}^{2}\right) \text {. ICNIRP }\end{array}$ \\
\hline $\begin{array}{l}\text { A 1 metro de } \\
\text { distancia del frente } \\
\text { del aplicador lumbar }\end{array}$ & 0,7 & $0,5-2$ & 10 & 50 \\
\hline $\begin{array}{l}\text { A 25 cm de la } \\
\text { parte trasera del } \\
\text { aplicador }\end{array}$ & 1,2 & $0,3-2$ & 10 & 50 \\
\hline $\begin{array}{l}\text { A 5 cm alrededor } \\
\text { de la cubierta del } \\
\text { equipo }\end{array}$ & 0,7 & $0,4-2$ & 10 & 50 \\
\hline $\begin{array}{l}\text { A 5 cm a lo largo } \\
\text { del cable coaxial }\end{array}$ & 1,6 & $0,5-2$ & 10 & 50 \\
\hline
\end{tabular}

El Instituto Nacional de Seguridad e Higiene en el Trabajo, con fecha 24/4/2006 y en el seno de un estudio de investigación sobre una metodología de evaluación sistemática de los CEM, realizó una medición en banda ancha, de intensidad de campo eléctrico, para radiofrecuencias y microondas cuyos resultados se encuentran en porcentajes inferiores al $26 \%$, por debajo de los límites de exposición recogidos en la Directiva 2004 (tablas II y III). 
Tabla II. Valor máximo encontrado con los aparatos encendidos, sin pacientes.

\begin{tabular}{|c|c|l|c|}
\hline $\begin{array}{c}\text { Campo eléctrico } \\
(2468.1356 \mathrm{MHz})\end{array}$ & $\begin{array}{c}\text { Valor máximo } \\
\text { encontrado }\end{array}$ & \multicolumn{1}{|c|}{ Límites del ICNIRP } & $\begin{array}{c}\text { Porcentajes de } \\
\text { los límites }\end{array}$ \\
\hline $\mathrm{E}(\mathrm{V} / \mathrm{m})$ & $15,690 \mathrm{~V} / \mathrm{m}$ & $\begin{array}{l}\text { Para público: } 61 \mathrm{~V} / \mathrm{m} \\
\text { Para trabajadores: } 137 \mathrm{~V} / \mathrm{m}\end{array}$ & $\begin{array}{c}25,721 \% \\
11,452 \%\end{array}$ \\
\hline
\end{tabular}

Tabla III. Valor máximo encontrado con los aparatos encendidos, con pacientes.

\begin{tabular}{|c|c|l|c|}
\hline $\begin{array}{c}\text { Campo eléctrico } \\
(2466.7719 \mathrm{MHz})\end{array}$ & $\begin{array}{c}\text { Valor máximo } \\
\text { encontrado }\end{array}$ & \multicolumn{1}{|c|}{ Límites del ICNIRP } & $\begin{array}{c}\text { Porcentajes de } \\
\text { los límites }\end{array}$ \\
\hline $\mathrm{E}(\mathrm{V} / \mathrm{m})$ & $6,319 \mathrm{~V} / \mathrm{m}$ & $\begin{array}{l}\text { Para público: } 61 \mathrm{~V} / \mathrm{m} \\
\text { Para trabajadores: } 137 \mathrm{~V} / \mathrm{m}\end{array}$ & $\begin{array}{c}10,359 \% \\
4,612 \%\end{array}$ \\
\hline
\end{tabular}

\section{DISCUSIÓN}

Con los niveles de exposición medidos en este servicio y los conocimientos existentes en el momento actual sobre efectos biológicos de los CEM no se detectan exposiciones que supongan riesgo para la salud de sus trabajadores.

\section{BIBLIOGRAFÍA}

(1) Directiva 2004/40/CE de 29 de abril de 2004, del Parlamento Europeo y del Consejo sobre las disposiciones mínimas de seguridad y de salud relativas a la exposición de los trabajadores a los riesgos derivados de los agentes físicos

(2) Scientific Committee on Emerging and Newly Identified Health Risks (SCENIHR). European Commission Health \& Consumer Protection Directorate-General. Possible effects of Electromagnetic Fields (EMF) on Human Health, 19 julio 2006. http://ec.europa.eu/health/ph_risk/committees/04_scenihr/scenihr_cons_03_en.htm

(3) EMF-NET. http://www.jrc.cec.eu.int/emfnet/pubblications.cfm

(4) Organización Mundial de la Salud. Proyecto Internacional CEM. http://www.who.int/peh-emf/project/EMF_Project/es/index.html
Las mediciones realizadas no tienen validez como evaluación de riesgos del Servicio de Rehabilitación.
(5) WHO. Estableciendo un dialogo sobre los riesgos de los campos electromagneticos. RADIACIÓN Y SALUD AMBIENTAL DEPARTAMENTO DE PROTECCIÓN DEL AMBIENTE HUMANO. ORGANIZACIÓN MUNDIAL DE LA SALUD. GINEBRA SUIZA.2005. ISBN 924354571 X (Clasificación LC/NLM: QT 34)

(6) NIEHS. EMF RAPID. Questions and Answers. June $2002 \mathrm{http}: / /$ www.niehs.nih.gov/emfrapid

(7) Asociación Española Contra el Cáncer. Campos Electromagnéticos y Cáncer: Preguntas y Respuestas.. www.todocancer.org JN/ES/12/2004

(8) www.microwavenews.com

(9) Review of the Epidemiologic Literature on RF and Health - 2004. http://www.icnirp.de/downloads.htm.

(10) Miranda Mayordomo J L. Editor. Rehabilitación Médica. Madrid. Grupo Aula Médica, S. L. 2004. 